\title{
Electric field orientations in solution and enzyme active site revealed by a two-directional vibrational probe
}

\author{
Chu Zheng ${ }^{1,+}$, Yuezhi Mao ${ }^{1,+}$, Jacek Kozuch ${ }^{2}$, Austin O. Atsango ${ }^{1}$, Zhe $\mathrm{Ji}^{1}$, Thomas E. Markland ${ }^{1, *}$, \\ and Steven G. Boxer ${ }^{1, *}$ \\ 1. Department of Chemistry, Stanford University, Stanford, CA 94305, USA \\ 2. Experimental Molecular Biophysics, Department of Physics, Freie Univeresität Berlin, 14195 \\ Berlin, Germany \\ TThese authors contributed equally to this work. \\ *Correspondence to: tmarkland@stanford.edu; sboxer@stanford.edu
}

\section{Abstract}

The catalytic power of an electric field depends on its magnitude and orientation with respect to the reactive chemical species. Understanding and designing new catalysts for electrostatic catalysis thus requires methods to measure the electric field orientation and magnitude at the molecular scale. We demonstrate that electric field orientations can be extracted using a twodirectional vibrational probe by exploiting the vibrational Stark effect of both the $\mathrm{C}=\mathrm{O}$ and $\mathrm{C}-\mathrm{D}$ stretches of a deuterated aldehyde. Combining spectroscopy with molecular dynamics and electronic structure partitioning methods, we demonstrate that despite distinct polarities, solvents act similarly in their preference for electrostatically stabilizing large bond dipoles at the expense of destabilizing small ones. In contrast, we find that for an active site aldehyde inhibitor of liver alcohol dehydrogenase, the electric field orientation deviates markedly from that found in solvents, providing direct evidence for the fundamental difference between the electrostatic environments of solvents and a preorganized enzyme active site. 


\section{Main}

The physical basis for the remarkable proficiency and specificity of enzymes under mild physiological conditions has been widely debated. One essential contribution is electrostatic preorganization, ${ }^{1,2}$ the precise positioning of functional groups with charges and dipoles by the protein scaffold that preferentially stabilizes the transition state (TS) over the reactant state (RS) due to the electric field at the active site, leading to a decrease in the activation free energy barrier (Fig. 1a). This mechanism has been directly supported by studies of several model enzymes, ${ }^{2,3,4,5,6,7}$ among which a prime example is ketosteroid isomerase (KSI), 8,9 where a large electric field $(\sim-150 \mathrm{MV} / \mathrm{cm})$ exerted by functional groups at the active site was measured, leading to a $\sim 10^{5}$-fold rate acceleration and accounting for a large fraction of the reduction in the activation free energy compared with the same reaction in water. Electric fields can be measured by exploiting the vibrational Stark effect (VSE), where IR or Raman frequency shifts are related to the projection of the field on a particular bond. This is accomplished by using VSE probes that have been calibrated via a combination of vibrational Stark spectroscopy (VSS), vibrational solvatochromism, and molecular dynamics (MD) simulations. ${ }^{2,8,10,11}$ 
a

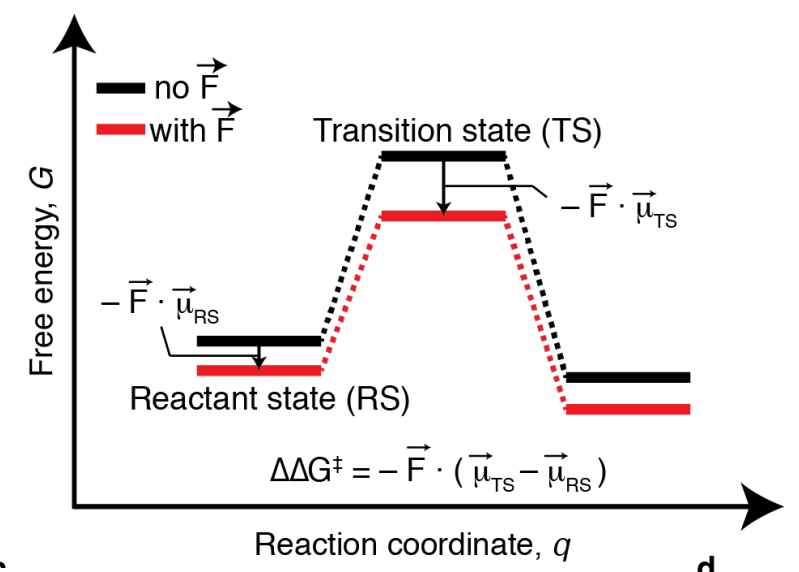

b

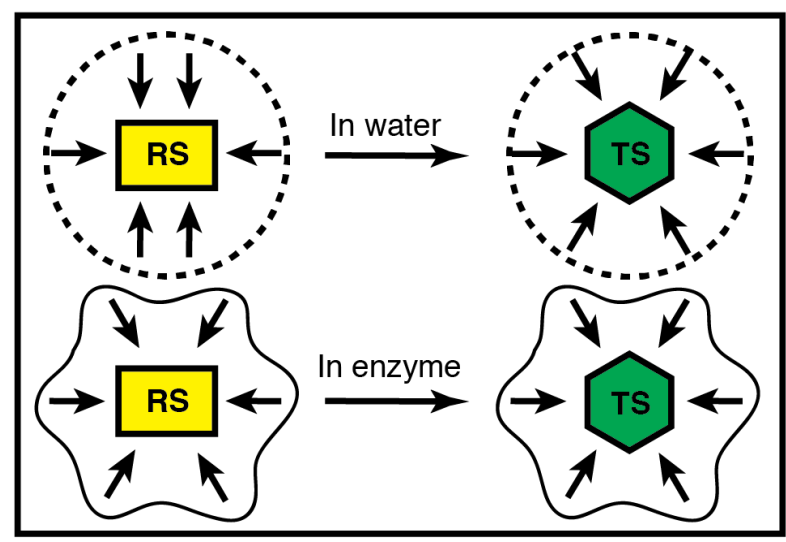

C
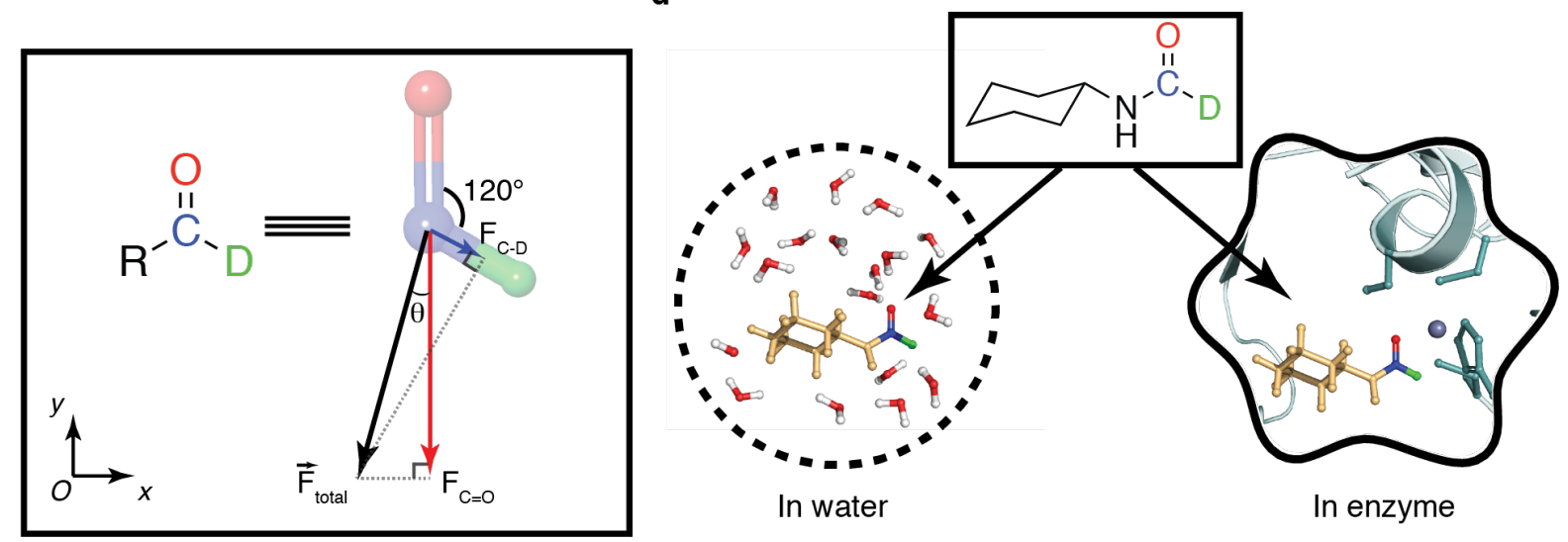

Fig. 1. Electrostatic catalysis and the two-directional vibrational probe used in this work (a) The model of electrostatic catalysis in enzymes: the reaction barrier is reduced by preferential stabilization of the TS because of the preorganized electric field, $\vec{F}$, at the active site. (b) Schematic illustration of the environmental response to the formation of the TS from RS during a chemical reaction that involves charge displacement (from rectangular to hexagonal for illustrative purposes). The top row illustrates the solvent reorganization in water (arrows represent the solvent dipoles), which contrasts with the minimal change in electric field orientation at the active site of enzymes due to electrostatic preorganization in the bottom row. (c) The concept of probing the orientation of an electric field by using the C=O and C-D stretch vibrations of an aldehyde. The total electric field on the two-dimensional plane of the aldehyde moiety is the vector sum of the fields measured along the two directions. (d) The structure of the VSE probe CXF-D used in this paper to measure electric field orientations in solvents (left) and at the active site of LADH (right). The crystal structure of the wild-type LADH in complex with NADH and CXF was resolved at $1.43 \AA$ (PDB: 7RM6).

The concept of electrostatic preorganization is illustrated in Fig. 1b, which compares a reaction involving charge reorganization occurring in water to that at the active site of an enzyme. 
Because solvent molecules, such as water, fluctuate around the reactant to stabilize the RS dipole moment on average, solvent configurations that stabilize the TS are rare and thus require substantial reorganization in the course of a reaction, a concept that forms the basis of electron and proton transfer theories such as the Marcus-Hush approach. ${ }^{12,13}$ In contrast, an electrostatically preorganized catalyst active site requires minimal adjustments to preferentially stabilize the charge distribution in the TS, thus minimizing the reorganization energy. ${ }^{1}$ Although this viewpoint has been supported by computational studies, $1,14,15,16,17,18,19,20,21$ there is limited direct experimental evidence for the critical assumption that the electric field orientation experienced by a substrate at enzyme active sites differs from that in solvents. The challenge to experimentally testing this hypothesis lies in the fact that most previous studies only measured the projection of the electric fields along one chemical bond at a time, and even when this is the bond involved in catalysis, it only provides information on the magnitude of the projected electric field but not the orientation of the total field.

In this work, we directly measure the orientation of electric fields in condensed-phase environments using a vibrational probe bearing two chemical bonds whose vibrational frequencies are both markedly shifted by the electric fields arising from their chemical environments, allowing us to measure the fields experienced by this probe along two directions (Fig. 1c). Using the deuterated (aldehyde H) form of $N$-cyclohexylformamide (CXF-D, Fig. 1d), a liver alcohol dehydrogenase (LADH) inhibitor, ${ }^{22,23}$ we obtain the projections of the electric field arising from solvents and the LADH active site on both the carbonyl $(C=O)$ and carbon-deuterium (C-D) bonds that are approximately $120^{\circ}$ from each other (Fig. 1C). Intriguingly, we find that the vibrational frequencies of $\mathrm{C}=\mathrm{O}\left(\bar{v}_{\mathrm{C}=\mathrm{O}}\right)$ and $\mathrm{C}-\mathrm{D}\left(\bar{v}_{\mathrm{C}-\mathrm{D}}\right)$ shift in opposite directions as the solvent 
polarity increases, indicating the coexistence of stabilization $(C=O)$ and destabilization $(C-D)$ of the chemical bonds around a single atom in solution. To elucidate these observations and introduce a field-frequency map for this probe in complex condensed phase environments, such as enzyme active sites, we develop a computational framework combining MD simulations with recently introduced electronic structure partitioning methods ${ }^{24,25}$ that gives improved accuracy and reliability in predicting the solvent electric fields compared to previously available methods. For a wide range of solvents, we observe a strong correlation between the electric fields experienced by the $\mathrm{C}=\mathrm{O}$ and $\mathrm{C}-\mathrm{D}$ bonds, suggesting that different solvents, despite their diverse chemical structures and polarities, exert electric fields of a similar orientation on the aldehyde moiety. By contrast, at the active site of LADH we reveal that the aldehyde moiety, which participates in the enzyme-catalyzed reaction, experiences a substantially different electric field orientation. This provides direct evidence for the intrinsic differences between the electrostatic environments of solvents and an enzyme active site experienced by a chemically relevant species.

\section{Results and discussion}

\section{Experimental validation of two-directional electric field measurements by vibrational}

Stark spectroscopy. The infrared absorption spectra for the C-D and C=O stretches in CXF-D are shown in Figs. $2 a$ and $2 b$, respectively. While the absorbance of the C-D stretch is typically $\sim 100$ times weaker than that of the $\mathrm{C}=\mathrm{O}\left(\right.$ or $\mathrm{C} \equiv \mathrm{N}$ ) stretch, ${ }^{26}$ the single $\mathrm{C}-\mathrm{D}$ stretch in CXF-D shows a reasonably large extinction coefficient $\left(\varepsilon_{\max } \approx 36 \mathrm{M}^{-1} \mathrm{~cm}^{-1}\right.$, which is about $1 / 30$ of that of $\left.\mathrm{C}=0\right)$, facilitating its application as a VSE probe. To validate the spectroscopic response of C=O and C-D in CXF-D to an external electric field, we used vibrational Stark spectroscopy (VSS), where an 
external electric field of known magnitude $(\sim 1.0 \mathrm{MV} / \mathrm{cm})$ was applied to the sample in a frozen glass (Fig. 2). The Stark spectrum is dominated by the second-derivative band shape from which the Stark tuning rates, $\left|\Delta \vec{\mu}_{\mathrm{c}=\mathrm{O}}\right| f_{\mathrm{C}=0}=1.13 \mathrm{~cm}^{-1} /(\mathrm{MV} / \mathrm{cm})$ and $\left|\Delta \vec{\mu}_{\mathrm{C}-\mathrm{D}}\right| f_{\mathrm{C}-\mathrm{D}}=1.29 \mathrm{~cm}^{-1} /(\mathrm{MV} / \mathrm{cm})$, are obtained (see Fig. S1 and Table S1 for details of fitting). The Stark tuning rate we obtain for the aldehyde carbonyl is in good agreement with the reported values for other carbonyl compounds, ${ }^{3,11}$ and the values for both $\mathrm{C}=\mathrm{O}$ and $\mathrm{C}-\mathrm{D}$ can be well reproduced $\left(1.01\right.$ and $1.22 \mathrm{~cm}^{-}$ $1 /(\mathrm{MV} / \mathrm{cm})$, respectively) using "Stark in silico" calculations, in which we performed ab initio frequency calculations with an external electric field applied along the bond directions while treating the solvent as a dielectric continuum (Supplementary Method S13, Text S3, Table S30). Here $f_{\mathrm{C}=\mathrm{O}}$ and $f_{\mathrm{C}-\mathrm{D}}$ are scalar approximations of the local field factor ${ }^{27}$ in the directions of the $\mathrm{C}=\mathrm{O}$ and C-D bonds. The local field factor is a tensor that describes the difference between the externally applied electric field and the actual electric field experienced by VSE probes and could be different for the $C=0$ and $C-D$ stretches ${ }^{10,27}$ (See Supplementary text S3). Although the sign of Stark tuning rates cannot be explicitly determined by VSS as the sample is isotropic, for vibrational transitions the Stark tuning rates should be positive in most cases because the excitedstate dipole moment is expected to be larger than that of the ground state due to vibrational anharmonicity. The dominance of the second-derivative band shape in the Stark spectra (Figs. 2c and $2 \mathrm{~d}$ ) indicates that the frequency shifts of both $C=O$ and $C-D$ stretch modes in response to an external electric field are primarily determined by the linear vibrational Stark effect ${ }^{28}$. Similar VSS results were also found for methyl formate-D, a different compound also carrying a deuterated aldehyde group (Fig. S2), demonstrating the generality of using a deuterated aldehyde to probe electric fields in two directions. 

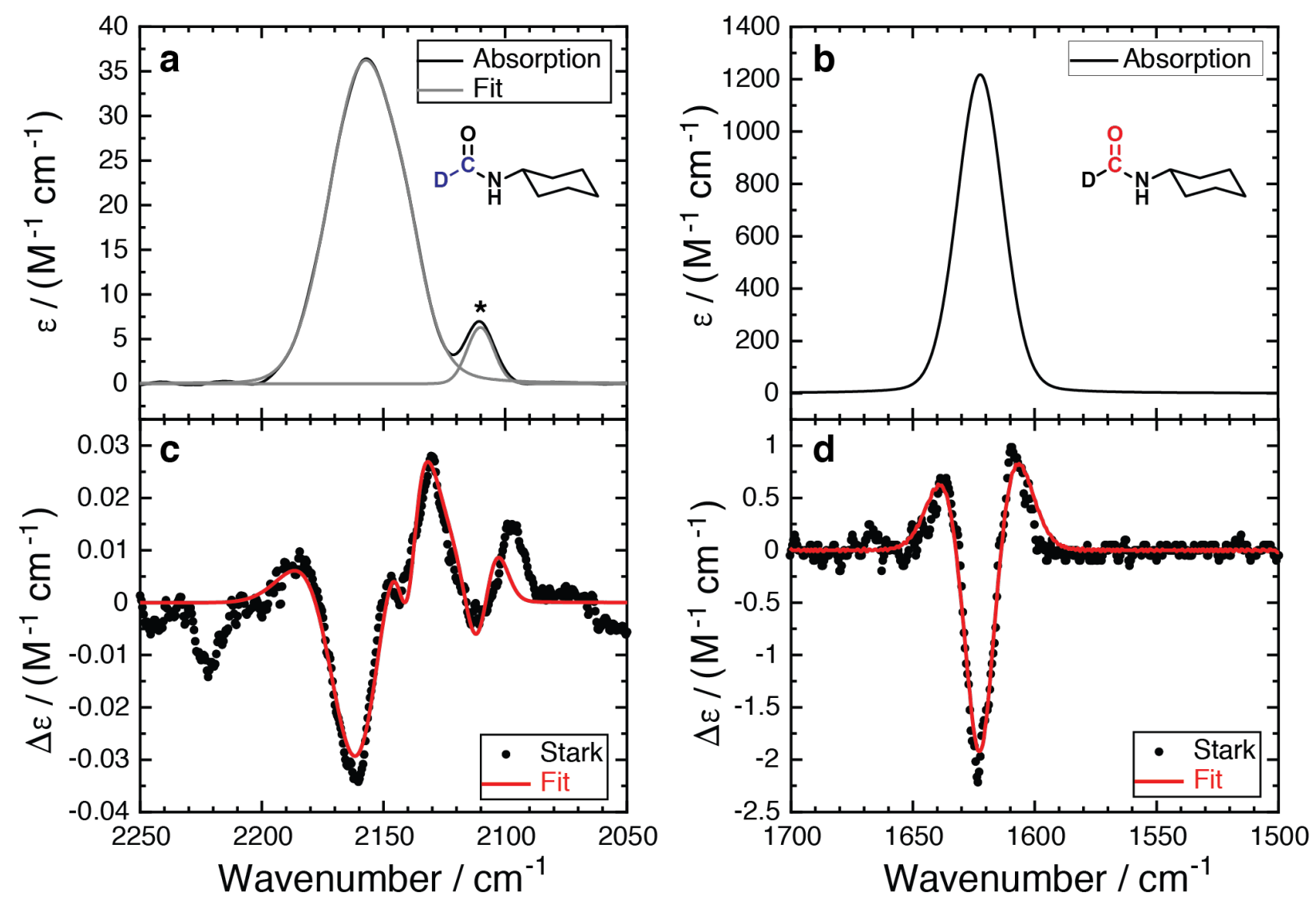

Fig. 2. Infrared absorption and Stark spectra of CXF-D at $77 \mathrm{~K}$ demonstrating the vibrational Stark effects of the C-D (left) and C=O (right) bonds. The upper panels $(a, b)$ are the infrared absorption spectra, and the lower panels (c, d) are the corresponding vibrational Stark spectra scaled to an applied field of $1.0 \mathrm{MV} / \mathrm{cm}$ with best fits (red lines, see Fig. S1 and Table S1 for the fitting details). The small shoulder peak labeled with an asterisk around $2100 \mathrm{~cm}^{-1}$ in (a) is likely due to a Fermi resonance. The C-D stretch was measured in 2-methyl tetrahydrofuran and the $\mathrm{C}=\mathrm{O}$ stretch mode in $\mathrm{D}_{2} \mathrm{O} /$ glycer(ol- $\left.\mathrm{D}_{3}\right)(\mathrm{v} / \mathrm{v} 1: 1)$. Different solvents were used because the $\mathrm{C}=\mathrm{O}$ stretch of CXF-D shows multiple bands in 2-methyl tetrahydrofuran, likely arising from dimerization due to intermolecular hydrogen bonds.

Opposite frequency shifts of $C=0$ and $C-D$ bonds upon solvation. Figure 3 shows that $\bar{v}_{\mathrm{C}=\mathrm{O}}$ and $\bar{v}_{\mathrm{C}-\mathrm{D}}$ exhibit frequency shifts in opposite directions upon increasing solvent polarity, a trend that is also observed for methyl formate-D (Fig. S3). While it has been widely observed that vibrational probes such as $\mathrm{C}=\mathrm{O}$ and $\mathrm{C} \equiv \mathrm{N}$ undergo redshifts with increasing solvent polarity, ${ }^{29}$ for this vibrational probe two bonds arising from the same atom show frequency shifts in opposite 
directions with C-D exhibiting a blueshift and $\mathrm{C}=\mathrm{O}$ a redshift. As shown below, this arises since solvent tends to organize around the more polar $\mathrm{C}=\mathrm{O}$ bond due to attractive electrostatic interactions resulting in a redshift, which in turn leads to destabilizing interactions sensed by the C-D bond at a $\sim 120^{\circ}$ angle from the $\mathrm{C}=\mathrm{O}$ direction.
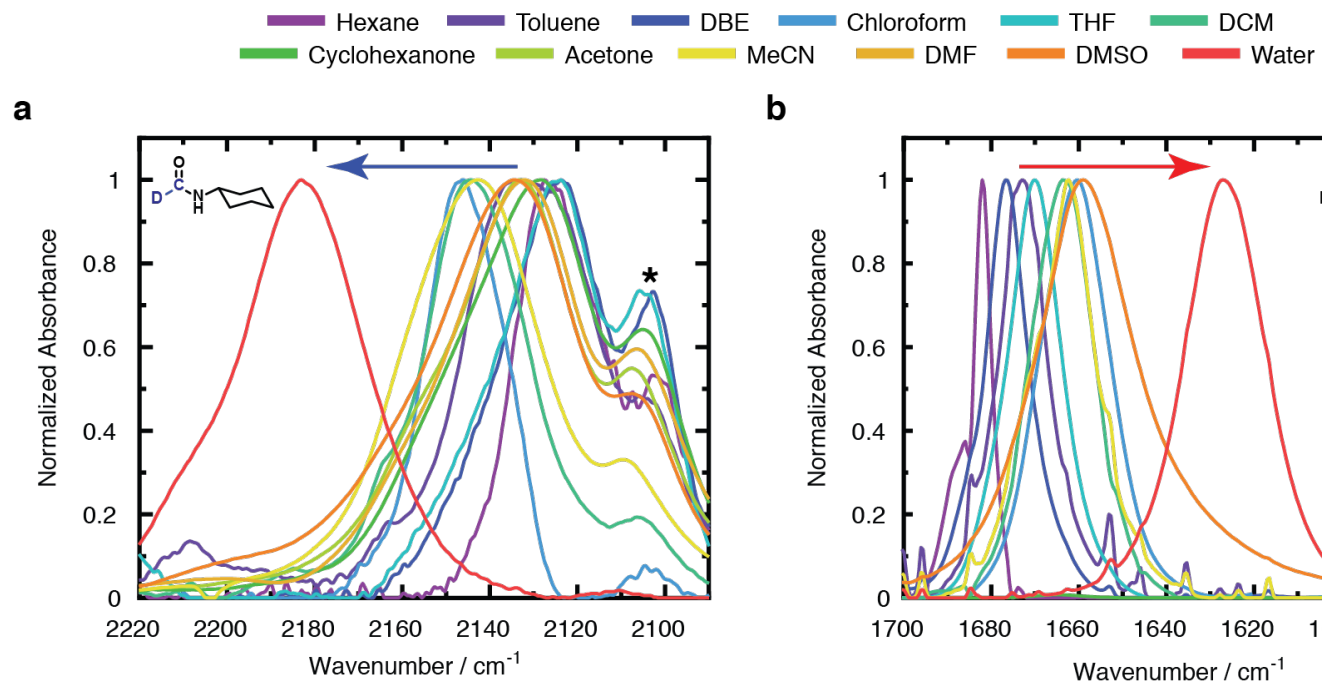

b

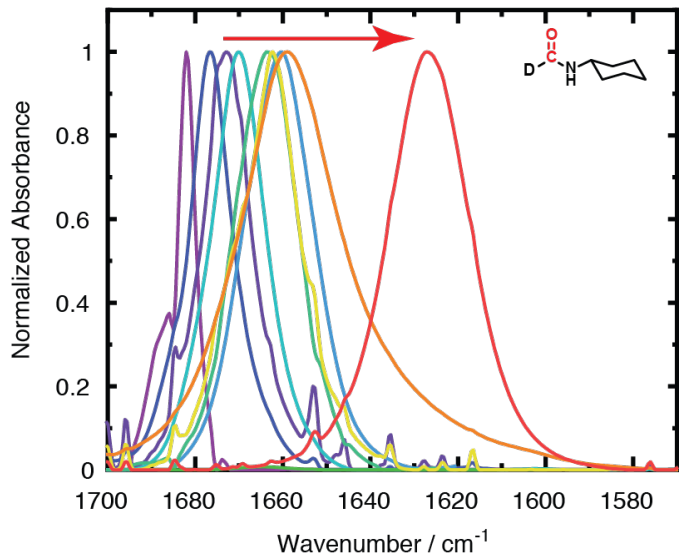

Fig. 3. Normalized infrared spectra of CXF-D in a series of solvents with various polarities demonstrating the solvatochromic shifts in the $C-D(a)$ and $C=O$ (b) frequencies. The concentrations of CXF-D in the measurements for $\bar{v}_{\mathrm{C}-\mathrm{D}}$ and $\bar{v}_{\mathrm{C}=\mathrm{O}}$ were $20 \mathrm{mM}$ and $1 \mathrm{mM}$, respectively. The blue and red arrows indicate the direction of frequency shifts of $C-D$ and $C=O$, respectively, upon increasing the solvent polarity. The small shoulder peak labeled with an asterisk around $2100 \mathrm{~cm}^{-1}$ in (a) is likely due to a Fermi resonance, which is evidenced by its minimal frequency shift with different solvents yet a more significant change in intensity as the C-D stretch band shifts.

\section{Evaluation of the solvent electric fields on $\mathrm{C}=\mathrm{O}$ and $\mathrm{C}-\mathrm{D}$ bonds using molecular}

simulation. To unravel the origins of this unusual solvatochromic behavior, we performed MD simulations and quantum mechanical (QM) electronic structure calculations to quantify the solvent electric fields projected on the $\mathrm{C}=\mathrm{O}$ and $\mathrm{C}-\mathrm{D}$ bonds. While fixed-charge $\mathrm{MD}$ simulations have been successfully used to establish field-frequency maps for $\mathrm{C}=\mathrm{O}$ in various compounds, $3,8,10,11,30$ the map produced for C-D using this method provides a poor correlation 
between the experimentally observed frequency shifts and the calculated fields (Fig. S4). To more accurately quantify the fields experienced by the C-D probe, we therefore adapted two recently developed electronic structure partitioning schemes ${ }^{24,25}$ to obtain more accurate solvent electric fields for configurations obtained from MD sampling. The QM-based approach can significantly improve the accuracy of field calculations because it avoids the usage of any parameters assigned by the force field, such as atomic charge or polarizability, but instead obtains the electric field directly from the electrostatic potential created by electrons and nuclei in the system. As shown in Tables S6-S12, the results obtained from both Subsystem Projected AO Decomposition $(\mathrm{SPADE})^{24}$ and Absolutely Localized Molecular Orbitals (ALMO) ${ }^{25}$ based partitioning schemes give consistent predictions of the solvent electric fields when combined with solvent configurations obtained from either fixed-charge ${ }^{11}$ or polarizable force fields. ${ }^{30,31}$ This approach thus provides a reliable way to quantify the solvent electric fields projected onto the vibrational probes, enabling us to uncover the physical origin of the unusual solvatochromic shifts as well as establishing the field-frequency map for CXF-D.

Figures $4 \mathrm{a}$ and $\mathrm{b}$ show that for both $\mathrm{C}-\mathrm{D}$ and $\mathrm{C}=\mathrm{O}$, over a wide range of solvent polarities (dielectric constants from 1 to 80 ), there is a strong linear correlation between the ensembleaveraged solvent electric fields obtained from our simulations and the experimentally measured frequencies. The ensemble-averaged solvent electric field along the $\mathrm{C}=\mathrm{O}$ bond is negative, which has also been found for many other carbonyl-containing molecules. ${ }^{3,11,30}$ The negative electric field along the $\mathrm{C}=\mathrm{O}$ bond signifies an attractive electrostatic interaction with solvents because the field is aligned with the bond dipole, which lies in the $\mathrm{O} \rightarrow \mathrm{C}$ direction. In contrast, the C-D bond in CXF-D has a more subtle dipole moment where both C and D are positively charged but 
$C$ is more so (Table S27) giving a dipole in the $D \rightarrow C$ direction. The electric field along the C-D bond is positive and thus anti-parallel with bond dipole direction, indicating that the electrostatic interaction with the solvent destabilizes the C-D covalent bond, consistent with the blueshift observed in its vibrational frequency as the solvent polarity increases. The correlation between the experimental frequency shifts and calculated fields thus provides strong support that the electrostatic interactions are responsible for modulating the vibrational frequencies. We note that the linearity of the correlation for C-D is not as good as that for $\mathrm{C}=\mathrm{O}\left(R^{2}=0.83\right.$ vs. 0.99$)$. This likely arises due to a Fermi resonance between the C-D stretch and the overtone of C-D bending modes that appears as a shoulder peak at around $2100 \mathrm{~cm}^{-1}$, consistent with previous studies on the vibrational spectra of aldehydes. ${ }^{32}$ To further validate our computational procedure, we also performed "Stark in silico" calculations in vacuum with an external electric field applied along the $\mathrm{C}=\mathrm{O}$ or $\mathrm{C}-\mathrm{D}$ direction, where we observed a strong correlation between the strength of the applied field and the computed C=O and C-D frequencies (Fig. S5, Table S14). 


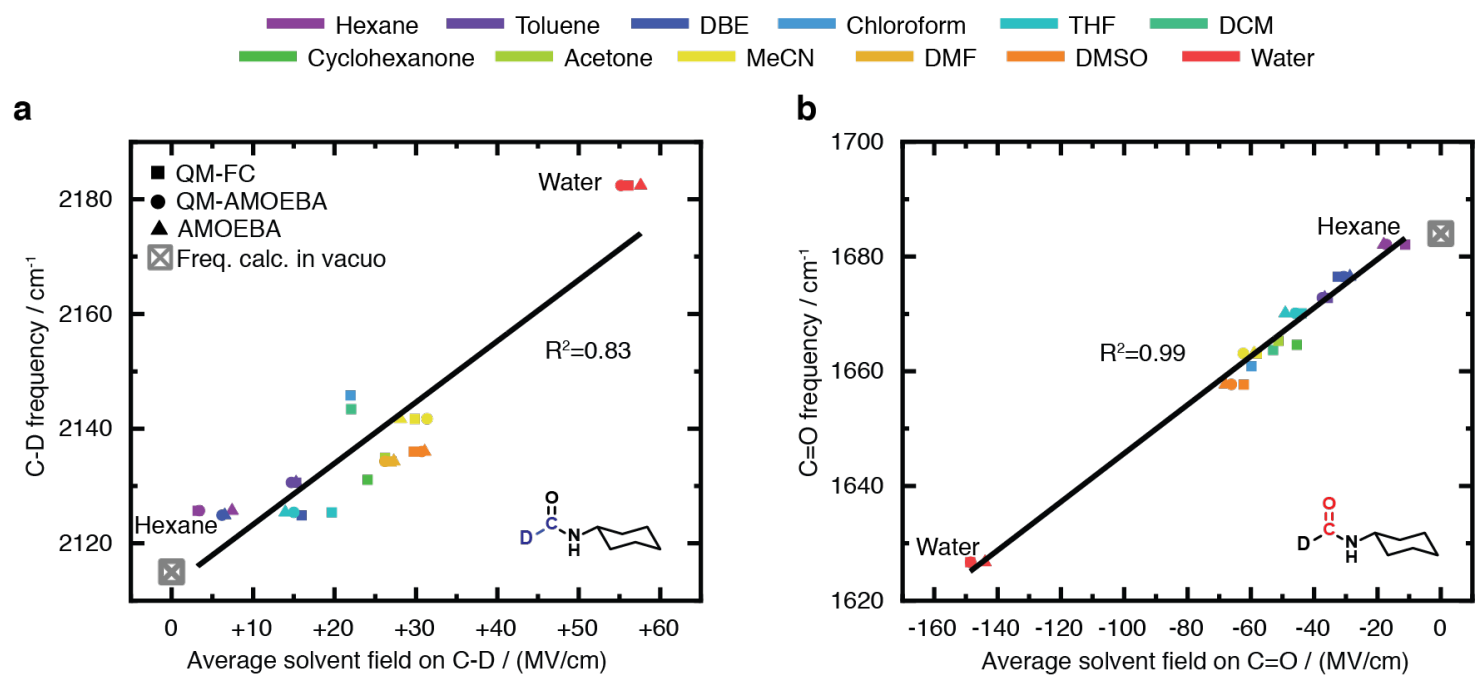

Fig. 4. Construction of the field-frequency maps for $C-D$ and $C=O$ in the CXF-D probe using experimentally measured frequency shifts and computed electric field projections along these bonds. Panels ( $a$ ) and ( $b$ ) show field-frequency correlations of C-D and C=O, respectively, plotted using the calculated solvent electric fields (ensemble average) and the experimentally measured peak frequencies. Electric fields obtained using three methods are shown and used for the leastsquares fits and additional results can be found in Tables S6-S13. "QM-FC" refers to using density functional theory and the SPADE electronic structure partitioning scheme ${ }^{24}$ (see SI Method Section) to calculate solvent electric fields based on fixed-charge MD trajectories. "QM-AMOEBA" refers to using the same QM method to calculate solvent electric fields while based on AMOEBA MD trajectories. "AMOEBA" refers to using the native parameters from the AMOEBA force field, such as the atomic permanent multipoles and polarizabilities, to calculate solvent electric fields. "Freq. calc. in vacuo" stands for the results of $a b$ initio harmonic frequency calculations of CXF-D in vacuo at the B3LYP/6-31+G(d,p) level (scaled by $0.964^{33}$ ), which serves as a cross-validation of the zero-field case and was not included in the fits. The least-squares regression lines based on the results of all three types of electric field calculations and the measured IR frequencies are $\bar{v}_{\mathrm{C}-\mathrm{D}}=1.07 F_{\mathrm{C}-\mathrm{D}}^{\text {solv }}+2112.61\left(R^{2}=0.83\right)$ and $\bar{v}_{\mathrm{C}=\mathrm{O}}=0.42 F_{\mathrm{C}=\mathrm{O}}^{\text {solv }}+1687.97\left(R^{2}=0.99\right)$ ， where $F_{\mathrm{C}-\mathrm{D}}^{\text {solv }}$ and $F_{\mathrm{C}=\mathrm{O}}^{\text {solv }}$ denote the projections of the solvent electric fields along the $\mathrm{C}-\mathrm{D}$ and $\mathrm{C}=\mathrm{O}$ directions, respectively.

The stark contrast in the solvent electric field directions on $\mathrm{C}=\mathrm{O}$ and $\mathrm{C}-\mathrm{D}$ manifests how these two bonds, connected to a common atom center, interact with the solvents. Since $\mathrm{C}=\mathrm{O}$ has a significantly larger bond dipole than C-D (Table S27), it is preferentially stabilized by the solvent, via both the local arrangements of solvent molecules and their electronic polarization. Due to the $\sim 120^{\circ}$ angle between the $\mathrm{C}=\mathrm{O}$ and $\mathrm{C}-\mathrm{D}$ bonds, the field induced by the solvent ordering around 
$C=O$ causes $C-D$ to experience a field that is in opposition to its bond dipole direction resulting in its destabilization. To further corroborate this physical picture, we performed simulations of $\mathrm{N}$ cyclohexylacetamide (Table S15), where the deuterium atom in CXF-D is replaced by a methyl group thus removing any specific interactions that may exist between C-D and solvent molecules. In this methylated analog we observe that the positive field along the $\mathrm{C}-\mathrm{C}$ (methyl) bond, which is in the same direction as C-D, is largely retained (reduced by $\sim 20 \%$ ), confirming that the positive electric field along C-D is a secondary effect due to the preferential stabilization of $C=O$. This picture of preferential stabilization of the more polar $\mathrm{C}=\mathrm{O}$ group provides additional details about the solute-solvent interactions that are not captured by the Onsager reaction field model, ${ }^{34}$ one of the most widely used models for solvation based on the concept that globally the molecular dipole of a solute experiences a stabilizing reaction field. Our results show clear evidence that locally, i.e., at the level of individual chemical bonds, ${ }^{35}$ there are both stabilizing and destabilizing electrostatic interactions experienced by solute molecules in solutions. Given its electrostatic origin, the trend in the opposite frequency shifts of $\mathrm{C}=\mathrm{O}$ and $\mathrm{C}-\mathrm{D}$ upon increasing solvent polarity can be qualitatively captured using a simple polarizable continuum model ${ }^{36}$ for the solvents (Table S16); however, a quantitative description of the solvatochromic effects necessitates considering the specific molecular ordering of the solvent around the probe.

To further elucidate the competition between the $C=O$ and $C-D$ dipoles in organizing the solvent around them, we examined the solvatochromic behavior of the carbonyl group in acetyl chloride where the C-D is replaced by the much more polar carbon-chlorine (C-Cl) bond. In this case, $\mathrm{C}-\mathrm{Cl}$ can much more effectively compete with $\mathrm{C}=\mathrm{O}$ for solvent organization, resulting in negative (stabilizing) electric fields along both bonds with the magnitude of that along $\mathrm{C}=\mathrm{O}$ 
reduced significantly (Fig. S12). Remarkably, we find that the $\mathrm{C}=\mathrm{O}$ of acetyl chloride shows only a $2-\mathrm{cm}^{-1}$ redshift going from hexane to acetonitrile (acetyl chloride is not stable in water), a much smaller solvent shift than that of CXF-D $\left(19 \mathrm{~cm}^{-1}\right)$ or any other carbonyl-containing molecule observed to date (Fig. S7, Table S17). Given that the C=O of acetyl chloride has a typical Stark tuning rate as obtained from both VSS experiments and "Stark in silico" calculations (Fig. S8, Table S1, Table S18), this extraordinarily small solvation redshift indicates that the $\mathrm{C}=\mathrm{O}$ of acetyl chloride, unlike that of CXF-D, gains a much lesser degree of electrostatic stabilization as the solvent polarity increases (see the Supplementary text $\mathrm{S} 1$ ). The observation that the $\mathrm{C}-\mathrm{Cl}$ bond suppresses the preferential stabilization of $\mathrm{C}=\mathrm{O}$ by solvent in acetyl chloride further underlines the ubiquity of distinct local solvation effects governed by bond dipoles, which may have farreaching implications in, for example, small-molecule catalyst design.

Probing the active-site electric field of LADH. LADH catalyzes a hydride transfer reaction, which is ubiquitous in many metabolic pathways, for the interconversion between alcohol and aldehyde using $\mathrm{NAD}^{+}$or $\mathrm{NADH}$ as a redox cofactor. ${ }^{37}$ To probe the magnitude and orientation of the electric fields in wild-type LADH, we introduced the CXF-D probe, which serves as a substrateanalog inhibitor, to its active site. As shown in Figs. $5 \mathrm{a} / \mathrm{b}$, this leads to red/blue-shifts in the $\mathrm{C}=\mathrm{O} / \mathrm{C}-\mathrm{D}$ frequencies that are even larger $\left(\bar{v}_{\mathrm{C}=\mathrm{O}}=1618 \mathrm{~cm}^{-1}, \bar{v}_{\mathrm{C}-\mathrm{D}}=2218 \mathrm{~cm}^{-1}\right)$ than those in water $\left(\bar{v}_{\mathrm{C}=\mathrm{O}}=1627 \mathrm{~cm}^{-1}, \bar{v}_{\mathrm{C}-\mathrm{D}}=2182 \mathrm{~cm}^{-1}\right)$, the most polar solvent that we investigated (Fig. S9, Table S19-S20). In addition, the linewidths of both C=O and C-D in the enzyme are much narrower than those in water, likely due to the smaller range of fluctuations possible in the activesite environment as has been observed in many enzymes. ${ }^{2}$ Based on the field-frequency map for CXF-D we obtained using a wide range of solvents (Fig. 4), the measured frequency shifts indicate 
that the active-site electric field projected on $\mathrm{C}=\mathrm{O}$ is $-166 \mathrm{MV} / \mathrm{cm}$, while that on $\mathrm{C}-\mathrm{D}$ is +99 $\mathrm{MV} / \mathrm{cm}$, which are both larger in magnitude than the average values observed in any of the solvents. CXF-D's $C=0$, similar to that of the substrate in aldehyde reduction reactions, is known to strongly interact with Ser-48 and $\mathrm{Zn}^{2+}$ at the active site $\mathrm{S2}^{22,23}$ (Fig. 1 and Fig. S10). Hence these specific interactions are the most likely sources of the large active-site electric field. In contrast, based on the crystal structure we obtained at $1.43 \AA$ (Fig. S10), the C-D in CXF-D does not interact strongly with the nearby residues, suggesting that the main contributions to its large positive electric field are from Ser-48 and $\mathrm{Zn}^{2+}$ that preferentially stabilize $\mathrm{C}=\mathrm{O}$ and destabilize $\mathrm{C}-\mathrm{D}$ as a secondary effect. 


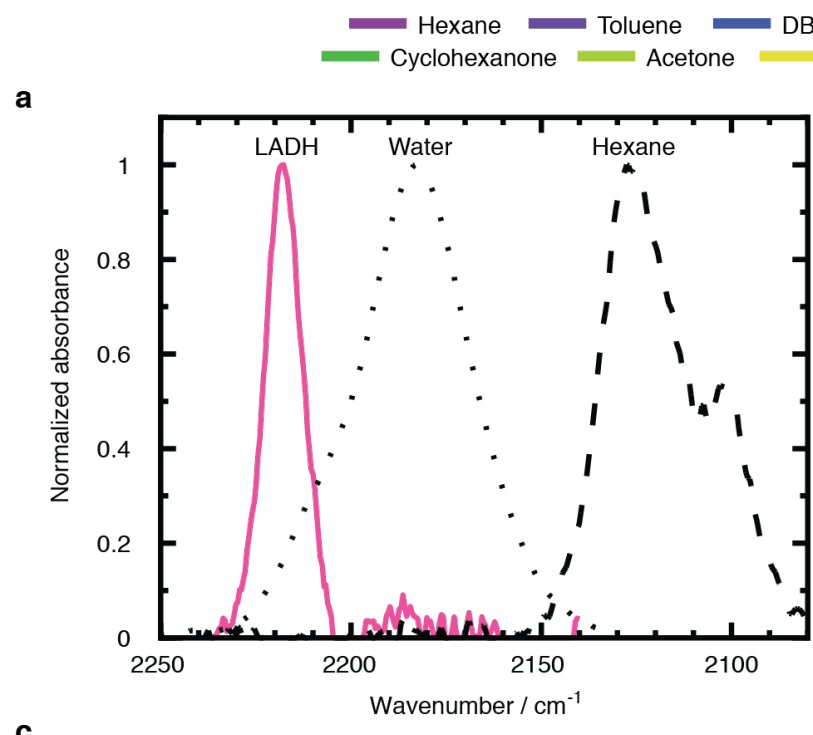

c
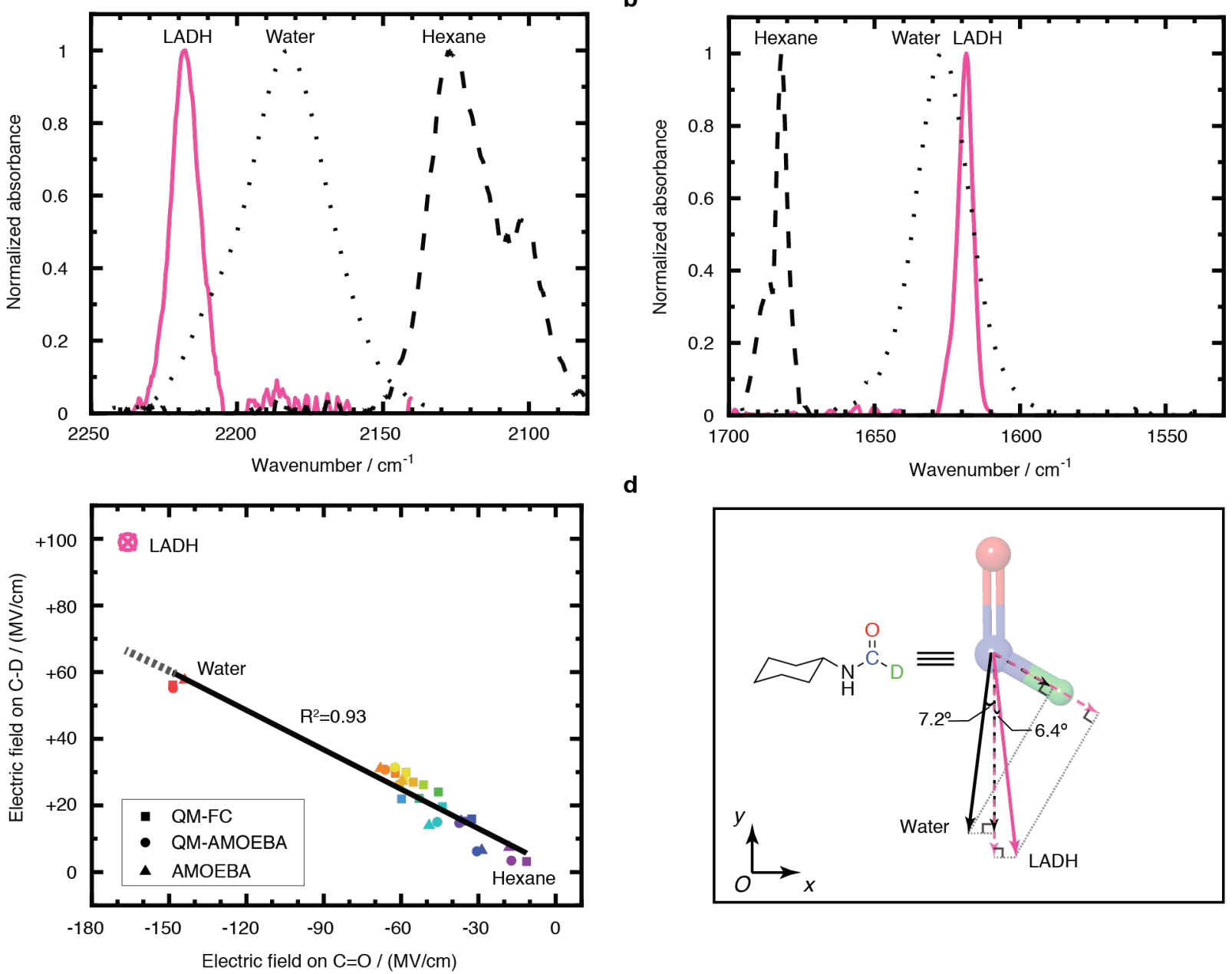

d

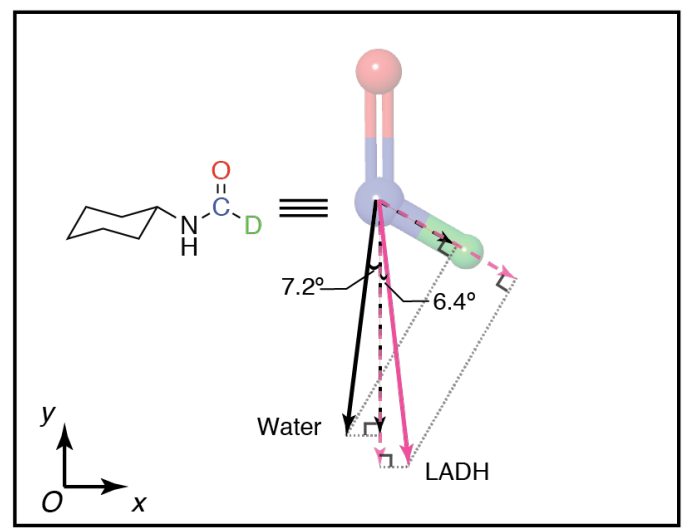

Fig. 5. Comparison of the electric field orientations in solvents and at LADH's active site revealed by two-directional electric field measurements. (a) and (b) are the normalized infrared spectra of $C-D$ and $C=O$, respectively, with CXF-D bound to the active site of wild-type LADH to form a ternary complex with NADH. The spectra of CXF-D in hexane and water are shown as dashed and dotted lines respectively for reference. (c) The correlation between the average electric fields on $C-D$ and $C=O$ in each solvent, using the color code given above, and LADH. The electric field calculations based on QM-FC, QM-AMOEBA, and AMOEBA are as defined in Fig. 4. The least-squares fit does not include the point for $\mathrm{LADH}$, and the result is $F_{\mathrm{C}-\mathrm{D}}=-0.39 F_{\mathrm{C}=0}+$ $1.21\left(R^{2}=0.93\right)$. (d) Visualization of the electric fields exerted by water (black arrow) and LADH (pink arrow) based on the results in panel (c). Dashed arrows represent the measured electric fields projected on $C=O$ and $C-D$, from which the orientations of the total electric field on the 2D plane of the aldehyde moiety were obtained (see Eq. 1). The solvents exert electric fields of different magnitudes but nearly the same direction: an angle of $\sim 7.2^{\circ}$ with respect to the $\mathrm{C}=\mathrm{O}$ dipole. In contrast, the active-site electric field of LADH has a larger magnitude and is oriented in a direction which differs from that in the solvents by $\sim 14^{\circ}$. 


\section{Comparison of the electric field orientation at the LADH active site to that in solvents.}

As shown in Fig. $5 c$, there is a strong linear correlation $\left(R^{2}=0.93\right)$ between the average electric fields projected on $\mathrm{C}=\mathrm{O}$ and $\mathrm{C}-\mathrm{D}$ for CXF-D in solvents. Despite the substantial changes in the electric field magnitudes across solvents due to their diverse polarities, the ratio between the average solvent electric field projected on $\mathrm{C}=\mathrm{O}\left(F_{\mathrm{C}=\mathrm{O}}\right)$ and $\mathrm{C}-\mathrm{D}\left(F_{\mathrm{C}-\mathrm{D}}\right)$ remains essentially unchanged at $F_{\mathrm{C}-\mathrm{D}} / F_{\mathrm{C}=\mathrm{O}}=-0.39$. Using this ratio and assuming that the angle between the $\mathrm{C}=\mathrm{O}$ and C-D groups is fixed at $120^{\circ}$ for the $\mathrm{sp}^{2}$ carbon we can extract the orientation of the electric field vector in the aldehyde plane, namely its angle to the bond dipole direction of $\mathrm{C}=\mathrm{O}$ that is labeled $\theta$ in Fig. 1c. This angle can be shown to be given by (see Supplementary text S2):

$$
\theta=\arctan \left[\frac{1}{\sqrt{3}}\left(1+2 \frac{F_{\mathrm{C}-\mathrm{D}}}{F_{\mathrm{C}=\mathrm{O}}}\right)\right]
$$

Therefore, the nearly constant value of $F_{\mathrm{C}-\mathrm{D}} / F_{\mathrm{C}=\mathrm{O}}$ across all the solvents indicates that the electric field orientations in the aldehyde plane are remarkably similar. This finding implies that, despite their distinct chemical structures and polarities, ${ }^{38}$ all these solvents tend to organize in a similar way to electrostatically stabilize the solute (vibrational probe). From the average value of this ratio we obtain an angle $\theta$ of $7.2^{\circ}$, i.e., the electric field is almost parallel to the $\mathrm{C}=\mathrm{O}$ bond dipole while pointing slightly away from C-D (Fig. $5 d$ ). This small value of $\theta$ further supports the physical picture of solvents preferentially stabilizing $\mathrm{C}=\mathrm{O}$, while the small deviation from being exactly parallel $\left(0^{\circ}\right)$ may be beneficial to stabilizing the solute globally by reducing the destabilization of C-D compared to a field that is perfectly parallel with the $\mathrm{C}=\mathrm{O}$ bond dipole.

In contrast, the electric field at the active site of LADH is oriented differently in the aldehyde plane as compared to that in solvents. Using the $F_{\mathrm{C}=0}$ and $F_{\mathrm{C}-\mathrm{D}}$ values for LADH (-166 and $+99 \mathrm{MV} / \mathrm{cm}$, respectively) and Eq. 1 , the angle $\theta$ obtained is $-6.4^{\circ}$. This angle corresponds 
to the electric field vector experienced by the aldehyde group of CXF-D being slightly skewed towards the opposite side of the $C=O$ bond dipole direction, i.e. towards $C-D$, compared to that in solvents where it is away from C-D (Fig. 5d). Hence in the two-dimensional (2D) plane of the aldehyde moiety the electric field orientation at the LADH active site is rotated by $\sim 14^{\circ}$ relative to that in solvents, leading to a field orientation that enhances the positive field projection along the C-D bond and thus further destabilizes it. This provides experimental evidence that unlike solvents, whose organization around the solute is largely directed by the solute bond dipoles to achieve global electrostatic stabilization, the enzyme active site is preorganized, tuning the relative stabilization of the aldehyde moiety's $C=O$ and $C-D$ bonds by imposing a distinct electric field on the reactive chemical moiety (the aldehyde group) that is rarely accessible in solvents in terms of both magnitude and orientation.

\section{Conclusions}

In summary, we have introduced an approach based on the VSE to measure both the magnitude and orientation of electric fields in condensed phase systems. By exploiting a twodirectional vibrational probe, CXF-D, we have shown that its deuterated aldehyde group can be calibrated using experiments and a computational framework based on molecular dynamics and electronic structure simulations to report the electric field strengths along both the $C=O$ and $C-D$ directions. Using this probe, we have demonstrated the coexistence of stabilizing and destabilizing electrostatic interactions experienced by the individual chemical bonds of a solute in a wide range of solvents, as well as the nearly invariant field orientation across solvents of distinct chemical structures and polarities. This provides direct experimental insights into how 
local solvent organization around a solute molecule driven by the difference in bond dipoles affords global electrostatic stabilization. In contrast, we have shown that the electric field at the active site of $L A D H$, as measured by the CXF-D probe, possesses a markedly different orientation that provides a greater electrostatic destabilization of the C-D bond than observed in solvents. Hence, while the electric field at an enzyme's active site and in aqueous solutions may be of comparable magnitudes, the orientations of these fields are intrinsically different. This supports the hypothesis that enzymes may provide preorganized electrostatic environments that maximize their catalytic power. ${ }^{1,2}$ Finally, the experimental and computational framework that we have introduced here to probe electric field orientations using deuterated aldehydes can be widely applied to other systems in complex environments and with complex solutes, including homogeneous catalysts, and thus serves as a prototype for characterization of electric field orientations to stimulate future investigations on the functional role of field orientation in enzymatic and electrostatic catalysis.

\section{Data availability}

All data supporting the finding of this study are available within this article and its supplementary information. The X-ray coordinates and structural factors have been deposited in the Protein Data Bank as entry 7RM6 for the wild-type enzyme in complex with NADH and Ncyclohexylformamide.

\section{Acknowledgements}

We would like to express our gratitude to Professor Bryce V. Plapp at University of lowa for providing detailed guidance regarding the expression and purification of liver alcohol 
dehydrogenase and much other valuable advice. We thank Dr. Chi-Yun Lin for his help on X-ray crystallography; Dr. Samuel Schneider, Jared Weaver, Jacob Kirsh, and Steven D. E. Fried for helpful discussions; Tom Carver at the Stanford Nano Shared Facilities for nickel coating on Stark windows; Theresa McLaughlin from Stanford University Mass Spectrometry (SUMS) for technical support of Mass Spectrometry. This work was supported in part by NIH Grant GM118044 (to S.G.B) and National Science Foundation Grant No. CHE-1652960 (to T.E.M). T.E.M. also acknowledges support from the Camille Dreyfus Teacher-Scholar Awards Program. This research also used resources of the National Energy Research Scientific Computing Center (NERSC), a U.S. Department of Energy Office of Science User Facility operated under Contract No. DE-AC0205CH11231, and the Sherlock cluster operated by the Stanford Research Computing Center.

\section{Author contributions}

C.Z. and S.G.B. designed the research. C.Z. performed the experiments and fixed-charge MD simulations. Y.M. developed the computational protocol combining MD simulations and QM calculations to quantify solvent electric field contributions, implemented the electronic structure partitioning schemes in the Q-Chem software package, and performed the QM calculations. J.K. performed the AMOEBA polarizable MD simulations. A.A. analyzed the data from MD simulations and wrote the codes to extract the truncated solute-solvent structures for the QM calculations. Z.J. synthesized $\mathrm{N}$-[formyl- $\left.{ }^{2} \mathrm{H}\right]$ cyclohexylformamide. C.Z., Y.M., T.E.M. and S.G.B. discussed results and wrote the manuscript. All authors contributed to improving the manuscript.

\section{Competing interests}

The authors declare no competing interests. 


\section{References}

1. Warshel A, Sharma PK, Kato M, Xiang Y, Liu H, Olsson MH. Electrostatic basis for enzyme catalysis. Chem Rev 2006, 106(8): 3210-3235.

2. Fried SD, Boxer SG. Electric Fields and Enzyme Catalysis. Annu Rev Biochem 2017, 86: 387-415.

3. Schneider SH, Boxer SG. Vibrational Stark Effects of Carbonyl Probes Applied to Reinterpret IR and Raman Data for Enzyme Inhibitors in Terms of Electric Fields at the Active Site. J Phys Chem B 2016, 120(36): 9672-9684.

4. Carey PR. Spectroscopic characterization of distortion in enzyme complexes. Chem Rev 2006, 106(8): 3043-3054.

5. Dong J, Lu X, Wei Y, Luo L, Dunaway-Mariano D, Carey PR. The strength of dehalogenase-substrate hydrogen bonding correlates with the rate of Meisenheimer intermediate formation. Biochemistry 2003, 42(31): 9482-9490.

6. Tonge PJ, Carey PR. Length of the acyl carbonyl bond in acyl-serine proteases correlates with reactivity. Biochemistry 1990, 29(48): 10723-10727.

7. Deng H, Zheng J, Clarke A, Holbrook JJ, Callender R, Burgner JW, 2nd. Source of catalysis in the lactate dehydrogenase system. Ground-state interactions in the enzyme-substrate complex. Biochemistry 1994, 33(8): 2297-2305.

8. Fried SD, Bagchi S, Boxer SG. Extreme electric fields power catalysis in the active site of ketosteroid isomerase. Science 2014, 346(6216): 1510-1514.

9. Wu Y, Boxer SG. A Critical Test of the Electrostatic Contribution to Catalysis with Noncanonical Amino Acids in Ketosteroid Isomerase. J Am Chem Soc 2016, 138(36): 11890-11895.

10. Fried SD, Boxer SG. Measuring electric fields and noncovalent interactions using the vibrational stark effect. Acc Chem Res 2015, 48(4): 998-1006. 
11. Fried SD, Bagchi S, Boxer SG. Measuring electrostatic fields in both hydrogen-bonding and non-hydrogen-bonding environments using carbonyl vibrational probes. J Am Chem Soc 2013, 135(30): 11181-11192.

12. Hush N. Adiabatic theory of outer sphere electron-transfer reactions in solution. Trans Faraday Soc 1961, 57: 557-580.

13. Marcus RA. On the theory of oxidation - reduction reactions involving electron transfer. I. J Chem Phys 1956, 24(5): 966-978.

14. Wu Y, Fried SD, Boxer SG. A Preorganized Electric Field Leads to Minimal Geometrical Reorientation in the Catalytic Reaction of Ketosteroid Isomerase. J Am Chem Soc 2020, 142(22): 9993-9998.

15. Vaissier Welborn V, Head-Gordon T. Computational Design of Synthetic Enzymes. Chem Rev 2019, 119(11): 6613-6630.

16. Welborn VV, Ruiz Pestana L, Head-Gordon T. Computational optimization of electric fields for better catalysis design. Nature Catalysis 2018, 1(9): 649-655.

17. Fuxreiter $M$, Mones $L$. The role of reorganization energy in rational enzyme design. Curr Opin Chem Biol 2014, 21: 34-41.

18. Kamerlin SC, Sharma PK, Chu ZT, Warshel A. Ketosteroid isomerase provides further support for the idea that enzymes work by electrostatic preorganization. Proc Natl Acad Sci U S A 2010, 107(9): 4075-4080.

19. Bím D, Alexandrova AN. Electrostatic regulation of blue copper sites. Chemical Science 2021.

20. Shaik S, Mandal D, Ramanan R. Oriented electric fields as future smart reagents in chemistry. Nat Chem 2016, 8(12): 1091-1098.

21. Xu L, Izgorodina El, Coote ML. Ordered Solvents and Ionic Liquids Can Be Harnessed for Electrostatic Catalysis. J Am Chem Soc 2020, 142(29): 12826-12833. 
22. Deng H, Schindler JF, Berst KB, Plapp BV, Callender R. A Raman spectroscopic characterization of bonding in the complex of horse liver alcohol dehydrogenase with NADH and N-cyclohexylformamide. Biochemistry 1998, 37(40): 14267-14278.

23. Ramaswamy S, Scholze M, Plapp BV. Binding of formamides to liver alcohol dehydrogenase. Biochemistry 1997, 36(12): 3522-3527.

24. Claudino D, Mayhall NJ. Automatic Partition of Orbital Spaces Based on Singular Value Decomposition in the Context of Embedding Theories. J Chem Theory Comput 2019, 15(2): 1053-1064.

25. Khaliullin RZ, Head-Gordon M, Bell AT. An efficient self-consistent field method for large systems of weakly interacting components. J Chem Phys 2006, 124(20): 204105.

26. Adhikary R, Zimmermann J, Romesberg FE. Transparent Window Vibrational Probes for the Characterization of Proteins With High Structural and Temporal Resolution. Chem Rev 2017, 117(3): 1927-1969.

27. Bublitz GU, Boxer SG. Stark spectroscopy: applications in chemistry, biology, and materials science. Annu Rev Phys Chem 1997, 48: 213-242.

28. Boxer SG. Stark realities. J Phys Chem B 2009, 113(10): 2972-2983.

29. Baiz CR, Blasiak B, Bredenbeck J, Cho M, Choi JH, Corcelli SA, et al. Vibrational Spectroscopic Map, Vibrational Spectroscopy, and Intermolecular Interaction. Chem Rev 2020, 120(15): 7152-7218.

30. Kozuch J, Schneider SH, Zheng C, Ji Z, Bradshaw RT, Boxer SG. Testing the Limitations of MD-Based Local Electric Fields Using the Vibrational Stark Effect in Solution: Penicillin G as a Test Case. J Phys Chem B 2021, 125(17): 4415-4427.

31. Fried SD, Wang LP, Boxer SG, Ren P, Pande VS. Calculations of the electric fields in liquid solutions. J Phys Chem B 2013, 117(50): 16236-16248.

32. Eggers DF, Lingren WE. C--H Vibrations in Aldehydes. Anal Chem 1956, 28(8): 1328-1329.

33. Johnson RD, III NIST Computational Chemistry Comparison and Benchmark Database, NIST Standard Reference Database Number 101, Release 21. August 2020 ed; 2020. 
34. Onsager L. Electric moments of molecules in liquids. J Am Chem Soc 1936, 58(8): 14861493.

35. Levinson NM, Fried SD, Boxer SG. Solvent-induced infrared frequency shifts in aromatic nitriles are quantitatively described by the vibrational Stark effect. J Phys Chem B 2012, 116(35): 10470-10476.

36. Tomasi J, Mennucci B, Cammi R. Quantum mechanical continuum solvation models. Chem Rev 2005, 105(8): 2999-3093.

37. Sekhar VC, Plapp BV. Rate constants for a mechanism including intermediates in the interconversion of ternary complexes by horse liver alcohol dehydrogenase.

Biochemistry 1990, 29(18): 4289-4295.

38. Reichardt C, Welton T. Solvents and solvent effects in organic chemistry. John Wiley \& Sons, 2011. 